\title{
Emerging measurements
}

\author{
H. Emons
}

Published online: 8 January 2013

(c) Springer-Verlag Berlin Heidelberg 2013

There are already many challenges in providing reliable results for established measurement tasks such as pesticide control in foodstuff or monitoring of tributyl tin in surface waters at the level of the EU environmental quality standard. But even more pronounced metrological challenges are created in emerging measurement areas such as the so-called key enabling technologies (KET). These are understood as knowledge and capital-intensive technologies which are associated with high research and development intensity, rapid and integrated innovation cycles, high capital expenditures and highly skilled employment. Nanotechnology, industrial biotechnology, advanced materials, photonics, micro- and nanoelectronics, and advanced manufacturing systems are classified by the European Commission as KETs. They are considered as main drivers for the development of future goods and services.

To advance, a technology requires measurement results for various purposes and KETs are no exception in this respect. Measurements are needed for developing a product, for establishing and surveying product and/or process specifications, for performing regulatory control, etc. However, KETs are characterized by much faster innovation cycles than traditional technology transfers from research and development $(\mathrm{R} \& \mathrm{D})$. That means the timing from basic research via technological research, product development and prototyping to manufacturing is shorter than usual. This creates additional metrological challenges: It is often not sufficiently known and understood which measurands (and their substitutes, respectively, see Accreditation and Quality Assurance (2012) 17:483) are the most relevant for decisions at early stages of the various

H. Emons ( $\square)$

Geel, Belgium

e-mail: JRC-IRMM-ACQUAL@ec.europa.eu innovation steps. For instance, which size parameter(s) have to be measured and controlled in the industrial process of integrating nanoparticles into textiles where the particles should exhibit antibacterial effects?

Moreover, the parameters of interest may rapidly change during the innovation cycle. This could lead to a situation that the required measurement methods (including crucial instrumentation or reagents) may not exist so far. Consequently, the measurement systems, including calibration as well as internal and external quality control measures and tools, have to be quickly adapted to moving targets. This is common for measurement activities in an R\&D environment and has been discussed in several ACQUAL publications. But manufacturing processes in very competitive markets have a different dimension of impact on society than R\&D. KET products are often on the market before measurement systems and tools are available for the parameters which demonstrate the existence and stability of the intended properties and prove that neither consumers nor the environment are at risk. Therefore, temporary solutions are frequently unavoidable. During such accelerated development phases, there is a tendency to neglect, or at least to pay only minor attention to, analytical quality assurance, thus increasing the danger of producing unreliable measurement data. Besides the risk of taking wrong decisions leading to wrong follow-up developments or misperceptions on the performance of the new product, one often observes that the omission of appropriate quality assurance measures is accepted also for longer application periods. The deficiency regarding quality assurance is becoming an 'established practice.' A common argument is the lack of validated and widely accepted measurement methods for the newly emerged task and the non-availability of corresponding reference materials and proficiency testing schemes. 
Indeed, there is an interrelation between the possibility to develop and validate a new measurement method and the availability of the necessary reference materials. New measurement tasks in emerging areas can only be based on adequate calibration and quality control systems (such as control charting, proficiency testing) which were developed in an iterative process. Therein, an initial measurement method is developed by using an initial batch of test material. This method is used to establish the homogeneity and stability of (another) material which serves for investigating method performance characteristics such as precision, selectivity and robustness. If the targeted quality criteria set by the future users are met, the method could be applied to characterize a material in view of obtaining a certified reference material (CRM). This first CRM would be then available for further specifying or modifying the initial measurement method.

In most cases, the measurands in such cycles are (at least partially) operationally defined (examples are discussed in the articles about the BIOREMA project published in this ACQUAL issue). Consequently, commonly accepted references, such as the way how the measurement shall be performed for addressing identical measurands, have to be established. For this purpose, the fast development and release of a documentary standard is often crucial for market acceptance of the new method. Such a documentary standard could be of only temporary use and would have to be accompanied by corresponding material measurement standards (reference materials). The challenge from KETs is coming from the need of having resources timely available for developing the standards on the one hand side and the likelihood of a limited applicability of both documentary and material standards on the other hand. They may only be valid for a short period of time in dependence on the overall speed of changes with respect to the measurand of interest.

Such temporary approaches have been and are also commonly used in more established measurement areas. There are many operationally (or method) defined parameters. Their existence and further use is usually taken for granted. However, their critical evaluation seems to be desirable on a case-by-case basis from a metrological point of view. The mini-series of articles on biofuel measurements published in this ACQUAL issue provide examples for the usefulness, but also for limitations of the longer term application of method-defined parameters and the metrological challenges to establish highest order reference materials as final anchor points for traceability chains or webs. Authors and readers of this journal are encouraged to report and discuss further experiences and approaches on the quality assurance of emerging measurements.

\section{Hendrik Emons}

Editor-in-Chief 